\title{
Elderly Deaf Patients' Health Care Experiences
}

\author{
Todd N. Witte, MD, and Anton J. Kuzel, MD, MHPE
}

Background: Approximately 10\% of the US population has some degree of hearing loss, and 2 million Americans are deaf. Most medical school curricula and major textbooks characterize deafness as pathologic condition only, which is at odds with the movement to understand the Deaf population as a minority group with a unique language and cultural tradition. Physicians might therefore be unprepared to meet the needs of deaf patients effectively and sensitively. This study seeks to understand the health care experiences of elderly Deaf adults in Richmond, Va.

Methods: The authors conducted focus groups of elderly Deaf persons. Real-time voice-interpretation of the sign language communication allowed for tape recording and full transcription. The authors independently analyzed the transcripts using an editing style, and incorporated feedback on their interpretation from participants.

Results: Participants experienced many practical barriers to effective health care, including problems with scheduling appointments and communicating with providers. They believed that providers are ill-prepared to care for them and worried that prejudice might be a more subtle obstacle. Participants seemed resigned to these circumstances.

Conclusions: The authors suggest a possible explanation for this perspective, and make specific recommendations for three levels of competency in caring for deaf patients. When the provider and the office staff provide methods to communicate with deaf patients using telephone-assisted communication, qualified interpreters, and some basic knowledge of lipreading or sign language, the care of deaf patients is greatly enhanced and the physician-patient relationship improved.(J Am Board Fam Pract 2000;13:17-22.)

Approximately $10 \%$ of the US population lives with some degree of hearing loss. Within this group is a subset of persons who are profoundly deaf. Deafness affects approximately 2 million Americans, for a prevalence of 1 in 100, making it likely a primary care physician will encounter a deaf person.,

Although these 2 million Americans share profound hearing losses, they are a heterogenous group and have a range of perspectives about their own deafness. ${ }^{3}$ Some members take a perspective of hearing loss as a pathologic condition and think of themselves as having dysfunctional ears. They tend to label themselves as deaf, hard-of-hearing, latedeafened, or hearing-impaired.

In contrast to this group are those who describe themselves as Deaf. The capitalization denotes that these persons do not consider their hearing loss a

Submitted, revised, 1 June 1999.

From the Virginia Commonwealth University School of Medicine (TNW), and the Department of Family Practice (AJK), Virginia Commonwealth University School of Medicine, Medical College of Virginia, Richmond. Address reprint requests to Anton Kuzel, MD, Department of Family Practice, MCV Campus, 1200 East Broad Street, PO Box 980251, Richmond, VA 23298-0251. disability, but rather a unique trait linking them to others like themselves, forming the basis for shared language, culture, and life experiences. ${ }^{4,5}$ In most communities in the United States, the language is a visual or signed language - in its most linguistically natural form, American Sign Language. ${ }^{6}$ This cultural group includes those with less than profound hearing losses who describe themselves as Deaf because they feel a greater psychosocial link to the culturally Deaf community than to the hearing majority. In this report we will use deaf to refer to a person with profound audiologic loss regardless of her or his group identity, and Deaf to refer to a member of the subset with a cultural affiliation. Hearing refers to a person without audiologic loss.

How does this diverse and distinct deaf population experience health care? Reportedly persons who are deaf and hard-of-hearing visit physicians more frequently, experience more difficulties in communicating with and understanding their physicians, have a lower comfort level with their physicians, and report lower subjective health status. ${ }^{7}$ It is also reported that there is a lack of knowledge and wide-spread misinformation on the part of physicians with respect to deaf patients. ${ }^{8}$ 
Despite the apparent gap in physicians' knowledge and abilities, there remains minimal attention to the deaf patient or the Deaf culture in medical education or in the classic adult patient generalist textbooks. ${ }^{9-13}$ The focus is instead on the pathophysiologic characteristics of deafness. It is not surprising that even the most well-intentioned medical student or physician might know little about this topic.

The size of the deaf community in the United States, its heterogeneity, and the limited attention to its health care needs within medical curricula raise many questions. How do members of the deaf community access health care? What is their experience? How do hearing physicians typically interact with deaf patients? What, if anything, is problematic about the experiences of deaf patients and the providers who care for them? What might be done to correct any problems?

\section{Methods}

Our questions suggest a program of study that should include individual and group interviews with Deaf patients and health care providers, as well as direct observations of interactions. ${ }^{14} \mathrm{We}$ selected a focus group format for our early work because it is well suited to initial exploration, and we recruited our study subjects from the elderly subpopulation of Deaf people in Richmond, Va. ${ }^{15,16}$ These persons were familiar with one another through their interactions with a local social and support organization, and they contributed their lifetime of health care experiences to the focus group. One of the authors who was familiar with the organization and its members recruited participants by face-to-face contact or by telephone using a teletypewriter ${ }^{\star}$ (TTY). Selection criteria included age 55 years or older, onset of hearing loss before age 10 years (mean age $=3$ years), involvement with Deaf culture, and use of sign language to communicate. The groups each included 6 to 8 participants.

*TTY is an abbreviation for teletypewriter. Also called a TDD, or telephone device for the deaf, it consists of an alphanumeric keypad and an LCD screen. It is used to send text messages back and forth by telephone. For hearing people who want to communicate by telephone with a deaf person but do not have access to a TTY, the toll-free state relay service is available whereby a third party operator acts as a go-between, relaying the spoken and typed messages.
One author served as facilitator and the other author as observer and co-facilitator. The organization director served as the sign language inter- $\frac{2}{2}$ preter, $\dagger$ voicing the communication of the Deaf participants in real-time.

Our questions, which came from the perspective of the author who was unfamiliar with the popula- $\stackrel{?}{+}$ tion, asked for descriptions of both typical and $\stackrel{\vec{\rho}}{\stackrel{5}{+}}$ memorable experiences. Both group interviews closed with a brainstorming session in which members suggested specific strategies for improving the $\frac{\widehat{\Phi}}{\square}$ quality of health care for Deaf patients. We gained is little new information from the second focus group $\overrightarrow{0}$ but heard much that supported the findings from $\omega$ the first. We therefore elected not to recruit a third focus group.

Tape recordings of the voiced sign language interpretation were fully transcribed by 1 author and were analyzed independently by both authors $\vec{v}$ using an editing approach. ${ }^{17} \mathrm{We}$ noted areas of 9 investigator agreement and explored differences to $\vec{c}$ determine a consensus interpretation. Focus group members' critiques of the authors' interpretations are reflected in this published report.

\section{Results}

Both groups provided detailed information about practical aspects of accessing and receiving routine health care. To begin, participants reported difficulty communicating with the physician's office by the telephone, whether to set up an appointment or receive results of tests. As do most deaf people, participants reported using a TTY to communicate by telephone.

Stories on this subject described physicians' offices that either had no TTY or did not know about the relay service. Participants also reported their 응 frustrations when the office telephoned without 9 using the TTY or relay service, and they were $\mathcal{N}$ unable to answer the call. Others commented on $\frac{D}{O}$ the need to rely on a hearing family member or friend to communicate by telephone with the physicians' offices: "The one thing that is disappointing is that they have to call my daughter first, and o then she informs me...." Some reported their $\stackrel{\mathbb{D}}{\mathscr{D}}$ dislike of the relay service, commenting that the relay operator sometimes types the hearing person's voiced words too fast. Many preferred that

†Certified by Registry of Interpreters for the Deaf. 
their physician's office have a TTY for direct oneto-one communication, to avoid the use of relay.

Another major area of discussion centered on communication in the office. Many described frustrating experiences in the waiting room. Despite informing the receptionist of their deafness, some of the participants were not informed it was their turn to see their physician because the staff relied on voice communication with them:

I go in and say, "Please let me know. I'll be sitting right over here...." But the receptionist often is just there calling out my name, and then when I don't respond, she'll move on to the next patient.

Participants also had much to express about communication with the physician. Receptively, the deaf patient can not hear the spoken word. Expressively, the Deaf patient typically communicates by sign language alone or by sign language coupled with speech. This combination makes for unique experiences:

... the doctor came over and tapped me on the shoulder. And he's mumbling and talking. And I said, "I'm Deaf..." And then he started yelling at me. And I said, "Hey, I'm Deaf. I can't hear at all!" And he kept on talking, and I kept on saying, "What???"

To overcome this communication barrier, Deaf patients and physicians have tried various techniques. The focus groups debated the desirability and effectiveness of lipreading, one of the more common approaches. Some participants reported learning to lip-read in school and accepted this mode of communication with their physicians as viable. These participants told stories of physicians speaking too fast, muttering, or having mustaches or beards that obscured their mouths, making lipreading difficult. Other participants, who learned sign language in school, took great offense to being asked to lip-read.

Viewpoints on writing back and forth varied, with some participants reporting no problems. Others reported some difficulty reading the physician's "big words" and questioned whether their messages were being properly understood by the physicians. Some experienced difficulty convincing physicians to write at all.

A final mode of communication uses a third person as an interpreter. In many cases participants used hearing family members or friends, noting that it was often difficult to coordinate appointment times with them. Confidence that the message was rendered faithfully was often compromised as well.

Another option is to use professional interpreters. Most participants believed that hospitals or physicians would not hire an interpreter for them, either because interpreters are scarce or because physicians are unwilling to pay the interpreter's fee. They reported frustration at having to postpone tests or at having to plan interpreter services nearly a week before the medical visit, which is not possible for acute care. Despite the overwhelming sentiment that "physicians won't pay for an interpreter," none of the participants reported ever directly asking their physician to hire or pay for an interpreter. Reasons cited for not making the request included fear of being refused, reluctance to upset the physician or physician-patient relationship, anticipation of the hiring of an unsatisfactory interpreter, and "I know he won't pay."

The concern about interpreter adequacy grew from participants' experiences with unskilled or unprofessional interpreters. Participants told of interpreters who were unable to understand their signing. They also worried that some of the physician's information was not relayed to them by the interpreter.

These practical barriers to care were sometimes coupled with fear or prejudice. According to one participant:

... [Doctors and nurses] are afraid of deaf people. I told the doctors to face me and I could see them shaking when they came in to take care of me. I said, 'I'm Deaf,'... and the doctor] throws up his hands like he doesn't know what to do... I don't think they feel comfortable.

Others punctuated their stories with comments like "just because he is deaf doesn't mean the doctor shouldn't do anything."

Despite the abundance of negative experiences expressed in the focus groups, we encountered unexpected patience and acceptance, which became evident as participants described experiences with less skilled interpreters: "[the interpreter] doesn't have to be perfect. At least they have some [signing skill], right?... I It's hard to learn 100\%." Another told a story about being in the emergency depart- 
ment and desperately wanting an interpreter but not being provided with one, concluding with, "I'm not complaining." One told of going to the same physician for 7 years, all the while dissatisfied with the care received.

Some participants expressed satisfaction with subspecialty care (ie, ophthalmology care, cardiology consultation) that used written communication on narrowly focused problems, but most of what we heard were stories of problems. Participants suggested several ways to improve health care for Deaf patients. Overall, they believed the medical community does not know enough about the nonpathologic aspects of deafness. Many asked that physicians learn about the psychosocial, cultural, and linguistic issues involved. Many suggested that health care professionals learn some form of nonverbal communication (eg, gesture, finger spelling, or sign language) sufficient for at least a basic conversation or assessment. They recommended that providers educate themselves further about issues of daily functioning, such as the use of TTYs, relay service, and closed captioning. Participants suggested rewarding providers with some sort of credit for the added training. Lastly, participants reported they had seen improvement in health care for Deaf patients, and some were optimistic that "in the future it will be better."

\section{Discussion}

The practical issues raised in this study are troubling. The long time spent in the waiting room and being bypassed for another patient are undesirable. Difficulty communicating by telephone with the physician's office adds further to patients' frustrations. These office barriers can engender negative feelings and decrease physician-patient rapport.

Our findings are in accord with those of previous studies in which deaf patients reported difficulty communicating with physicians. ${ }^{7,18,19}$ Physicians generally share the viewpoint of several of the participants that lipreading is an acceptable means of communicating in the medical setting. ${ }^{8}$ Studies, by contrast, indicate that $60 \%$ to $70 \%$ of sounds and words appear similar on the lips, leaving much of the comprehension dependent on guesswork. ${ }^{3}$ These statistics indicate that lipreading is not a highly effective means of communicating vital medical information.

Although our participants expressed some acceptance of written communication, research shows that perhaps because of the aural-oral natures of English, the literacy of deaf people is severa years behind their hearing counterparts, on average्ष्क at or below a fourth-grade reading level. ${ }^{20}$ Written?

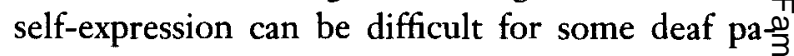
tients as well, and some are prone to writing En-0 glish in a style resembling American Sign Lan guage. For example, a Deaf patient might report leg. burt mine to convey pain in a leg. Thus, evenf among the elderly Deaf adults who are comfortableo with writing back and forth, effective physician $\frac{\overline{\bar{\rho}}}{\bar{\sigma}}$ patient communication is not guaranteed.

Interpreters are potentially more effective ats bridging the language barrier between English and $\overrightarrow{0}$ sign language. Our participants rarely experienced the use of a qualified, competent interpreter, how ever. One important repercussion of using a family member, friend, or an inadequately skilled signer is that this person might filter the communicationconveyed to the patient or physician. ${ }^{21}$ Issues of $\vec{v}$ privacy and confidentiality also arise when involv-? ing a third party who has no ethical obligations. Furthermore, the skill of an uncertified interpreter? is questionable. Even with the best intentions, fam ily members, friends, Good Samaritans in the hospital, or professional interpreters with limited? training and experience might have expressive oro receptive language limitations, especially in challenging medical contexts. ${ }^{22,23}$ Such scenarios risk ineffective communication and an ethical breach. $\stackrel{\square}{2}$

Several of our elderly Deaf participants believed $\vec{\Xi}$ they were treated as different or less than equal, yet they seemed resigned to the prejudice and practicalo problems. This attitude could be a functional re-ş sponse to circumstances beyond their control. It might also be that we, the authors, are interested in promoting social activism, and we are therefore more sensitive to these issues. The history of the deaf population, which has experienced paternalism응 and oppression by the hearing majority, however, $N$ might be another contributing factor to their ap- $\frac{\vec{O}}{2}$ parent resignation. ${ }^{24}$ The cultural perspective on deafness did not gain broad attention until rela- స్ల tively recently with the "Deaf President Now!" protest of 1988 , and the elderly generation represented by our participants might have been less $\stackrel{\mathbb{\Phi}}{\rightarrow}$ affected by this civil rights movement. ${ }^{25}$

The health care problems of our participants $\frac{\overrightarrow{0}}{\overrightarrow{0}}$ have legal implications, particularly in the era of $\frac{0}{\mathbb{Q}}$ the Americans with Disabilities Act (ADA), and Deaf people are winning lawsuits against hospitals 
and physicians who are in violation of this act. ${ }^{26-28}$ There are also compelling ethical reasons to remedy these problems if Deaf patients' claims to autonomy, justice, and beneficence are inadequately met by current medical practice. ${ }^{9}$

Regardless of the motivation, providers can make specific changes to improve health care services for Deaf patients. ${ }^{18}$ Health care providers should use TTYs and the relay service to improve telephone communication. They should be aware of the limitations of written communication. If communicating by writing, providers should avoid medical jargon and complex sentences or vocabulary, as well as abbreviated content. Providers should also know that written expression from a Deaf patient might stray from conventional English and might require additional questions to understand the intended meaning. They should remember that the patient's literacy and writing skill "is a misleading indicator of education or intelligence." 3 Providers should acknowledge the inefficiency and limitations of lipreading. It is imperative to verify that key information is accurately understood even with patients who prefer to lip-read.

The best way to communicate is by using sign language or sign language coupled with speech, the preference of most Deaf patients. The benefits of true provider-patient rapport, which might be established by a sign-language-proficient provider, are indisputable. A popular misconception, however, is that sign language is easy to learn, and beginners are often falsely confident in their skills. If not truly conversant, the provider should be cautious in using sign language, because the patient's history can be misinterpreted, and the physician risks conveying misinformation.

Providers not proficient in sign language can use an interpreter who is receptively and expressively skilled. The best way to ensure accurate communication is to use only interpreters with either advanced state-awarded interpreting certificates indicating their preparedness for medical interpreting or certification by reputable national agencies, such as Registry of Interpreters for the Deaf. By virtue of their code of ethics, certified interpreters are obliged to uphold professional standards and patient confidentiality. ${ }^{29} \mathrm{We}$ believe they are the preferred choice to assist health professionals, and we strongly discourage the use of uncertified interpreters, including friends or family members of the patient. Lastly, providers can encourage elderly
Deaf patients to bring up their own concerns by maintaining an atmosphere of unhurried respect.

We suggest the following three levels of competency in health care services to Deaf patients:

Level 1 - Provider is aware of Deaf culture and the heterogeneity of the deaf community; office incorporates and is trained in the use of TTY or relay service or both. Provider and office write in an appropriate manner and verify both their own and the patient's understanding. Provider and office have sought out interpreters who are qualified. The services of health professionals who have a greater competency have been found and are used.

Level 2 - Meets criteria of level 1, plus the provider has a more in-depth understanding of Deaf culture. Provider and office staff are competent in basic gesture and finger spelling.

Level 3 - Meets criteria of level 2, plus provider communicates with deaf patient in their preferred mode, including sign language, by either learning and achieving competency in sign language, or employing a qualified and appropriately certified interpreter.

Personnel at a given facility will choose their level based on their own interests, the number of deaf patients in their practice, and the availability of resources. To achieve these competencies, providers should seek assistance from consultants or local or national associations and should institute appropriate curricula at all levels of professional education. We believe that with little cost or effort, all facilities are capable of meeting level 1 criteria.

There currently are providers, some of whom are deaf, who satisfy level 3 criteria. Such practices have shown greater patient satisfaction and improved preventive care outcomes. ${ }^{18}$ One could therefore speculate that if other factors were the same, a deaf patient would prefer a level 3 provider to one of a lesser competency. It is impractical, however, to set this as a goal for all communities, and the participants in our study would clearly appreciate even level 1 competency.

Our investigation provides some insight into the health care experiences of 14 elderly Deaf adults in Richmond, Va. Although we used several strategies to ensure the quality of our study, we recognize that our study is limited not only by the size and specificity of the sample group, but also by the attention to stories of care rather than observation of actual care. We acknowledge that persons from different localities; persons of different ages with 
different ages of onset of hearing loss, degrees of hearing loss, and preferred communication modes; and persons who are affiliated with the Deaf culture would have varying experiences. Our aim in this study is not to generalize our findings, but to sensitize ourselves and our colleagues in the health professions to the needs of Deaf patients in our local communities.

The authors wish to thank: the deaf community of Richmond, especially the elderly members who generously gave of their time and wisdom; Commonwealth Catholic Charities of Richmond and the Community Center for the Deaf and Hard of Hearing; the MCV Foundation (Department of Family Practice); and Sarah L. Booth, Mable Heiskill, and Ellen Trimble.

\section{References}

1. Adams PF, Hardy AM. Current estimates from the national health interview survey: United States 1988. Hyattsville, Md: National Center for Health Statistics (Vital and health statistics: Series 10, Data from the national health survey, no. 173), 1989. [DHHS publication no (PHS) 89-1501.]

2. Benderly BL. Dancing without music. Washington, DC: Gallaudet University Press, 1980.

3. Issues to consider in deaf and hard-of-hearing patients. The Committee on Disabilities of the Group for the Advancement of Psychiatry. Am Fam Physician 1997;56:2057-64, 2067-8.

4. Dolnick E. Deafness as culture. Atlantic Monthly 1993;272(3):37-40, 43, 46-8, 50-3.

5. Erting $C$, Woodward J. sign language and the deaf community. Discourse Processes 1979;2:198-300.

6. Lucas C, Valli C. When Is ASL?. In: Snider BD, Erting CJ, Johnson RC, Smith DL, editors. The deaf way: perspectives from the International Conference on Deaf Culture. Washington, DC: Gallaudet University Press 1994:356-64.

7. Zazove P, Niemann LC, Gorenflo DW, et al. The health status and health care utilization of deaf and hard-of-hearing persons. Arch Fam Med 1993;2:745-52.

8. Ebert DA, Heckerling PS. Communication with deaf patients. Knowledge, beliefs, and practices of physicians. JAMA 1995;273:227-9.

9. Zazove P, Doukas, DJ. The silent health care crisis: ethical reflections of health care for deaf and hardof-hearing persons. Fam Med 1994;26:387-90.

10. Fauci AS, Braunwald E, Isselbacher KJ, et al, editors. Harrison's principles of internal medicine. 14th ed. New York: McGraw-Hill, 1998.

11. Bennett JC, Plum F, editors. Cecil textbook of medicine. 20th ed. Philadelphia: WB Saunders, 1996.
12. Rakel RE, editor. Textbook of family practice. 5 th $\frac{c}{3}$ ed. Philadelphia: WB Saunders, 1995.

13. Taylor RB, editor. Family medicine: principles and practice, 5th ed. New York: Springer, 1998.

14. Morse JM. Designing funded qualitative research. In: Denzin NK, Lincoln YS, editors. Handbook of qualitative research. Thousand Oaks, Calif: Sage Publications, 1994.

15. Borkan JM. Conducting qualitative research in the $\stackrel{\overrightarrow{\vec{D}}}{\stackrel{\vec{m}}{+}}$ practice setting. In: Bass MJ, et al, editors. Conducting research in the practice setting. Newbury Park, $\frac{\bar{\sigma}}{\bar{\omega}}$ Calif: Sage Publications, 1993.

16. Kuzel AJ. Sampling in qualitative inquiry. In: Crabtree BF, Miller WL, editors. Doing qualitative research. 2nd ed. Newbury Park, Calif: Sage Publications, 1999.

17. Miller WL, Crabtree BF. Primary care research: a multimethod typology and qualitative road map. In: $\frac{O}{F}$ Crabtree BF, Miller WL, editors. Doing qualitative? research. 2nd ed. Newbury Park, Calif: Sage Publi- $\vec{\omega}$ cations, 1999.

18. MacKinney TG, Walters D, Bird GL, Nattinger AB. 을 Improvements in preventive care and communication for deaf patients: results of a novel primary health care program. J Gen Intern Med 1995;10: 133-7.

19. Schein JD, Delk MT. Survey of health care for deaf $N$ people. The Deaf American 1980;32(5):5-6, 27.

20. Paul PV. Literacy and deafness. Boston: Allyn and Bacon, 1998.

21. Orr RD. Treating patients from other cultures. Am Fam Physician 1996;53:2004-6.

22. Cokely D. The interpreted medical interview: it loses something in the translation. The Reflector $\frac{\text { 의 }}{3}$ 1982;3:5-10.

23. Baker DW, Parker RM, Williams MV, Coates WC, Pitkin K. Use and effectiveness of interpreters in an emergency department. JAMA 1996;275:783-8.

24. Lane $H$. The mask of benevolence: disabling the deaf community. New York: Alfred A Knopf, 1992.

25. Gannon JR. The week the world heard Gallaudet. 응 Washington, DC: Gallaudet University Press, 1989. 윽

26. Naiman $v$ New York University, No. 95 Civ. 6469- N LMM, 1997 WL 249970 (S;D:N.Y. May 13, 1997). D

27. Lamonica $v$ North Shore University Hospital \& Soutbside Hospital. 95-CIV-4993-LDW (EDNY). US Dis- 응 trict Court, Eastern District of New York.

28. Eldridge $v$ British Columbia (Attorney General), File no. 24896. April 24, 1997; October 1997:9.

29. Humphrey JH, Alcorn BJ. So you want to be an interpreter: an introduction to sign language interpreting. Amarillo, Tex: H\&H Publishing, 1994. 